\title{
Higher-order q-derivatives and their applications to subclasses of multivalent Janowski type $q$-starlike functions
}

\author{
Bilal Khan' ${ }^{1}$ Zhi-Guo Liu' ${ }^{1}$, H.M. Srivastava ${ }^{2,3,4}$, Serkan Araci ${ }^{5 *}$ (D, Nazar Khan ${ }^{6}$ and Qazi Zahoor Ahmad
}

"Correspondence:
serkan.araci@hku.edu.tr
${ }^{5}$ Department of Economics, Faculty
of Economics Administrative and
Social Sciences, Hasan Kalyoncu
University, TR-27410 Gaziantep,
Turkey
Full list of author information is
available at the end of the article

\begin{abstract}
In the present investigation, with the help of certain higher-order $q$-derivatives, some new subclasses of multivalent $q$-starlike functions which are associated with the Janowski functions are defined. Then, certain interesting results, for example, radius problems and the results related to distortion, are derived. We also derive a sufficient condition and certain coefficient inequalities for our defined function classes. Some known consequences related to this subject are also highlighted. Finally, the well-demonstrated fact about the $(p, q)$-variations is also given in the concluding section
\end{abstract}

MSC: Primary 30C45; 30C50; 30C80; secondary 11B65; 47B38

Keywords: Analytic functions; Multivalent functions; $q$-Difference (or q-Derivative) operator; q-Starlike functions; Distortion theorems; Janowski functions; Radius problem; Coefficient inequalities

\section{Introduction, motivation and definitions}

The class of multivalent or ( $\mathfrak{p}$-valent) functions in

$$
\mathbb{U}=\{z: z \in \mathbb{C} \text { and }|z|<1\}
$$

with series representation

$$
f(z)=z^{\mathfrak{p}}+\sum_{n=1}^{\infty} a_{n+\mathfrak{p}} z^{n+\mathfrak{p}} \quad(\mathfrak{p} \in \mathbb{N}=\{1,2,3, \ldots\}),
$$

is denoted here by $\mathcal{A}(\mathfrak{p})$. We note that

$$
\mathcal{A}(1):=\mathcal{A} \text {. }
$$

(c) The Author(s) 2021. This article is licensed under a Creative Commons Attribution 4.0 International License, which permits use, sharing, adaptation, distribution and reproduction in any medium or format, as long as you give appropriate credit to the original author(s) and the source, provide a link to the Creative Commons licence, and indicate if changes were made. The images or other third party material in this article are included in the article's Creative Commons licence, unless indicated otherwise in a credit line to the material. If material is not included in the article's Creative Commons licence and your intended use is not permitted by statutory regulation or exceeds the permitted use, you will need to obtain permission directly from the copyright holder. To view a copy of this licence, visit http://creativecommons.org/licenses/by/4.0/. 
Moreover, the class of multivalent or (p-valent) starlike functions is denoted by $\mathcal{S}^{*}(\mathfrak{p})$, which consists of functions $f \in \mathcal{A}(\mathfrak{p})$ that satisfy the following condition:

$$
\Re\left(\frac{z f^{\prime}(z)}{f(z)}\right)>0 \quad(\forall z \in \mathbb{U}) .
$$

It is easy to see that

$$
\mathcal{S}^{*}(1)=\mathcal{S}^{*},
$$

where by $\mathcal{S}^{*}$ we denote the class of starlike functions in the open unit disk $\mathbb{U}$.

We next recall that the class $\mathcal{S}^{*}$ of starlike functions was generalized by Janowski [7] as follows.

Definition 1 ([7]) A function $f \in \mathcal{A}$ is said to belong to the class $\mathcal{S}^{*}[X, L]$ if and only if

$$
\Re\left(\frac{(L-1)\left(\frac{z f^{\prime}(z)}{f(z)}\right)-(X-1)}{(L+1)\left(\frac{z f^{\prime}(z)}{f(z)}\right)-(X+1)}\right) \geqq 0 \quad(-1 \leqq L<X \leqq 1) .
$$

In order to present, we adopt the following notations and definitions. Throughout this article we assume that

$$
0<q<1 \quad \text { and } \mathfrak{p} \in \mathbb{N}=\{1,2,3, \ldots\}
$$

Definition 2 For $0<q<1$, we define the $q$-number $[\lambda]_{q}$ by

$$
[\lambda]_{q}= \begin{cases}\frac{1-q^{\lambda}}{1-q} & (\lambda \in \mathbb{C} \backslash\{0\}), \\ \sum_{k=0}^{j-1} q^{k}=1+q+q^{2}+\cdots+q^{j-1} & (\lambda=j \in \mathbb{N}), \\ 0 & (\lambda=0) .\end{cases}
$$

Definition 3 The $q$-factorial $[j]_{q} ! q$-factorial is defined as follows:

$$
[0]_{q} !:=1 \text { and }[j]_{q} !=\prod_{k=1}^{j}[k]_{q} .
$$

It is easy to see from Definition 2 and Definition 3 that

$$
\lim _{q \rightarrow 1-}[j]_{q}=j \text { and } \quad \lim _{q \rightarrow 1-}[j]_{q} !=j !
$$

Definition 4 For $f \in \mathcal{A}$, the $q$-difference (or the $q$-derivative) operator $\mathfrak{D}_{q}$ in a given subset of the set $\mathbb{C}$ of complex numbers is defined by (see [5] and [6])

$$
\left(\mathfrak{D}_{q} f\right)(z)= \begin{cases}\frac{f(z)-f(q z)}{(1-q) z} & (z \neq 0) \\ f^{\prime}(0) & (z=0)\end{cases}
$$

provided that $f^{\prime}(0)$ exists. 
We can easily see from (1.2) that

$$
\lim _{q \rightarrow 1-}\left(\mathfrak{D}_{q} f\right)(z)=\lim _{q \rightarrow 1-} \frac{f(z)-f(q z)}{(1-q) z}=f^{\prime}(z)
$$

for a differentiable function $f$ in a given subset of $\mathbb{C}$. Furthermore, from (1.1) and (1.2) we obtain

$$
\begin{aligned}
& \left(\mathfrak{D}_{q}^{(1)} f\right)(z)=[\mathfrak{p}]_{q} z^{\mathfrak{p}-1}+\sum_{n=1}^{\infty}[n+\mathfrak{p}]_{q} a_{n+\mathfrak{p}} z^{n+\mathfrak{p}-1}, \\
& \left(\mathfrak{D}_{q}^{(2)} f\right)(z)=[\mathfrak{p}]_{q}[\mathfrak{p}-1]_{q} z^{\mathfrak{p}-2}+\sum_{n=1}^{\infty}[n+\mathfrak{p}]_{q}[n+\mathfrak{p}-1]_{q} a_{n+\mathfrak{p}} z^{n+\mathfrak{p}-2}, \\
& \left(\mathfrak{D}_{q}^{(\mathfrak{p})} f\right)(z)=[\mathfrak{p}]_{q} !+\sum_{n=1}^{\infty} \frac{[n+\mathfrak{p}]_{q} !}{[n]_{q} !} a_{n+\mathfrak{p}} z^{n},
\end{aligned}
$$

where $\left(\mathfrak{D}_{q}^{(\mathfrak{p})} f\right)(z)$ denotes the $q$-derivative of the function $f(z)$ of order $\mathfrak{p}(\mathfrak{p} \in \mathbb{N})$.

Now, for each function $f$ of the class $\mathcal{A}(\mathfrak{p})$, the expression in (1.1) when differentiated $s$ times with respect to $z$ yields

$$
\left(\mathfrak{D}_{q}^{(s)} f\right)(z)=\frac{[\mathfrak{p}]_{q} !}{[\mathfrak{p}-s]_{q} !} z^{\mathfrak{p}-s}+\sum_{n=1}^{\infty} \frac{[n+\mathfrak{p}]_{q} !}{[n+\mathfrak{p}-s]_{q} !} a_{n+\mathfrak{p}} z^{n+\mathfrak{p}-s} .
$$

The branch of $q$-calculus has many applications in various branches of mathematics and physics. Also, the operator $\mathfrak{D}_{q}$ ( $q$-derivative operator) has some remarkable and distinctive applications, which makes it significant. Ismail et al. [4] were the first who made use of the $q$-derivative operator and starlike functions and defined a new class $\mathcal{S}^{*}$ of $q$-starlike functions. However, initially, it was Srivastava [24] who used the basic (or q-) hypergeometric functions

$$
{ }_{\mathfrak{r}} \Phi_{\mathfrak{s}} \quad\left(\mathfrak{r}, \mathfrak{s} \in \mathbb{N}_{0}=\{0,1,2, \ldots\}=\mathbb{N} \cup\{0\}\right)
$$

in geometric function theory (GFT) of complex analysis (see, for details, [24]). Very recently, Srivastava's published review article [25] gave another flavor to this subject. In his published review article [25], Srivastava highlighted the triviality of the so-called $(p, q)$ calculus.

The aforementioned works of Ismail et al. [4] and Srivastava [24, 25] motivated a number of mathematicians to give their findings. In the above-cited work by Srivastava [25], many well-known convolution and fractional $q$-operators were surveyed. For example, Wongsaijai and Sukantamala [35] studied certain subclasses of $q$-starlike functions from different viewpoints and prospectives. In particular, they studied various coefficient inequalities, inclusion properties, and sufficient conditions. Furthermore, the work of Wongsaijai and 
Sukantamala [35] was systematically generalized by Srivastava et al. [33]. In fact, by making use of the $q$-calculus and the Janowski functions, Srivastava et al. (see [33, 34]) defined three new subclasses of $q$-starlike functions. Several other authors (see, for example, $[16,18,20,22,29,30])$ studied and generalized the classes of $q$-starlike from different viewpoints and prospectives. For some more recent investigation about $q$-calculus and fractional calculus in geometric functions theory and in other branches of mathematics and physics, we may refer the interested reader to $[1-3,11-15,17,19,21,26,27,31,32,36,37]$. In this paper, we shall be concerned mainly with generalizations of the works presented in $[8,33]$, and [35].

Definition 5 (see [4]) A function $f \in \mathcal{A}$ is in the functional class $\mathcal{S}_{q}^{*}$ if

$$
f(0)=f^{\prime}(0)-1=0
$$

and

$$
\left|\frac{z}{f(z)}\left(\mathfrak{D}_{q} f\right) z-\frac{1}{1-q}\right| \leqq \frac{1}{1-q} .
$$

By means of some higher-order $q$-derivatives, the following subclasses of multivalent $q$-starlike functions, which are associated with the Janowski functions, are defined below.

Definition 6 A multivalent function $f$ of the class $\mathcal{A}(\mathfrak{p})$ is in the class $\mathcal{S}_{(q, 1)}^{*}[\mathfrak{p}, v, s, X, L]$ if

$$
\Re\left(\frac{(L-1) \frac{z^{\nu}\left(\mathfrak{D}_{q}^{(\nu+s)} f\right)(z)}{\left(\mathfrak{D}_{q}^{(s)} f\right)(z)}-(X-1)}{(L+1) \frac{z^{\nu}\left(\mathcal{D}_{q}^{(\nu+s)} f\right)(z)}{\left.\mathfrak{D}_{q}^{(s)} f\right)(z)}-(X+1)}\right) \geqq 0 .
$$

We call $\mathcal{S}_{(q, 1)}^{*}[\mathfrak{p}, v, s, X, L]$ the class of multivalent higher-order $q$-starlike functions of the first type involving Janowski functions.

Definition 7 A multivalent function $f$ of the class $\mathcal{A}(\mathfrak{p})$ is in the class $\mathcal{S}_{(q, 2)}^{*}[\mathfrak{p}, v, s, X, L]$ if

$$
\left|\frac{(L-1) \frac{z^{\nu}\left(\mathfrak{D}_{q}^{(v+s)} f\right)(z)}{\left(\mathfrak{D}_{q}^{(s)} f\right)(z)}-(X-1)}{(L+1) \frac{z^{v}\left(\mathfrak{D}_{q}^{(v+s)} f\right)(z)}{\left(\mathfrak{D}_{q}^{(s)} f\right)(z)}-(X+1)}-\frac{1}{1-q}\right|<\frac{1}{1-q} .
$$

We call $\mathcal{S}_{(q, 2)}^{*}[\mathfrak{p}, v, s, X, L]$ the class of multivalent higher-order $q$-starlike functions of the second type involving Janowski functions.

Definition 8 A multivalent function $f$ of the class $\mathcal{A}(\mathfrak{p})$ is in the class $\mathcal{S}_{(q, 3)}^{*}[\mathfrak{p}, v, s, X, L]$ if

$$
\left|\frac{(L-1) \frac{z^{\nu}\left(\mathfrak{D}_{q}^{(\nu+s)} f\right)(z)}{\left(\mathfrak{D}_{q}^{(s)} f\right)(z)}-(X-1)}{(L+1) \frac{z^{\nu}\left(\mathfrak{D}_{q}^{(\nu+s)} f\right)(z)}{\left(\mathcal{D}_{q}^{(s)} f\right)(z)}-(X+1)}-1\right|<1 .
$$

We call $\mathcal{S}_{(q, 3)}^{*}[\mathfrak{p}, v, s, X, L]$ the class of multivalent higher-order $q$-starlike functions of the third type involving Janowski functions. 
Remark 1 First of all, it is easy to see that, for $(0 \leqq \alpha<1)$,

$$
\begin{aligned}
& \mathcal{S}_{(q, 1)}^{*}[1,1,0,(1-2 \alpha),-1]=\mathcal{S}_{(q, 1)}^{*}(\alpha), \\
& \mathcal{S}_{(q, 2)}^{*}[1,1,0,(1-2 \alpha),-1]=\mathcal{S}_{(q, 2)}^{*}(\alpha),
\end{aligned}
$$

and

$$
\mathcal{S}_{(q, 3)}^{*}[1,1,0,(1-2 \alpha),-1]=\mathcal{S}_{(q, 3)}^{*}(\alpha),
$$

where the function classes

$$
\mathcal{S}_{(q, 1)}^{*}(\alpha), \quad \mathcal{S}_{(q, 2)}^{*}(\alpha), \quad \text { and } \quad \mathcal{S}_{(q, 3)}^{*}(\alpha)
$$

were studied by Wongsaijai and Sukantamala [35]. Secondly, we have

$$
\begin{aligned}
& \mathcal{S}_{(q, 1)}^{*}[1,1,0, X, L]=\mathcal{S}_{(q, 1)}^{*}[X, L], \\
& \mathcal{S}_{(q, 2)}^{*}[1,1,0, X, L]=\mathcal{S}_{(q, 2)}^{*}[X, L],
\end{aligned}
$$

and

$$
\mathcal{S}_{(q, 3)}^{*}[1,1,0, X, L]=\mathcal{S}_{(q, 3)}^{*}[X, L]
$$

where the function classes

$$
\mathcal{S}_{(q, 1)}^{*}[X, L], \quad \mathcal{S}_{(q, 2)}^{*}[X, L], \quad \text { and } \quad \mathcal{S}_{(q, 3)}^{*}[X, L],
$$

were studied by Srivastava et al. [33]. Thirdly, for $(0 \leqq \alpha<1)$, we have

$$
\begin{aligned}
& \mathcal{S}_{(q, 1)}^{*}[\mathfrak{p}, v, s,(1-2 \alpha),-1]=\mathcal{S}_{(q, 1)}^{*}(\mathfrak{p}, v, s, \alpha), \\
& \mathcal{S}_{(q, 2)}^{*}[\mathfrak{p}, v, s,(1-2 \alpha),-1]=\mathcal{S}_{(q, 2)}^{*}(\mathfrak{p}, v, s, \alpha),
\end{aligned}
$$

and

$$
\mathcal{S}_{(q, 3)}^{*}[\mathfrak{p}, v, s,(1-2 \alpha),-1]=\mathcal{S}_{(q, 3)}^{*}(\mathfrak{p}, v, s, \alpha),
$$

where the function classes

$$
\mathcal{S}_{(q, 1)}^{*}(\mathfrak{p}, v, s, \alpha), \quad \mathcal{S}_{(q, 2)}^{*}(\mathfrak{p}, \nu, s, \alpha), \quad \text { and } \quad \mathcal{S}_{(q, 3)}^{*}(\mathfrak{p}, v, s, \alpha),
$$

were studied by Khan et al. [8].

In this paper, many properties and characteristics such as, for example, sufficient conditions, inclusion results, distortion theorems, and radius problems are investigated. We also indicate relevant connections of our results with those in a number of other related works on this subject. 


\section{Main results}

We start here by giving an inclusion results for the classes

$$
\mathcal{S}_{(q, 1)}^{*}[\mathfrak{p}, v, s, X, L], \quad \mathcal{S}_{(q, 2)}^{*}[\mathfrak{p}, v, s, X, L], \quad \text { and } \quad \mathcal{S}_{(q, 3)}^{*}[\mathfrak{p}, v, s, X, L]
$$

of the generalized multivalent $q$-starlike function classes, which involve the Janowski functions.

Theorem 1 If $-1 \leqq L<X \leqq 1$, then

$$
\mathcal{S}_{(q, 3)}^{*}[\mathfrak{p}, v, s, X, L] \subset \mathcal{S}_{(q, 2)}^{*}[\mathfrak{p}, v, s, X, L] \subset \mathcal{S}_{(q, 1)}^{*}[\mathfrak{p}, v, s, X, L]
$$

Proof Firstly, we let $f \in \mathcal{S}_{(q, 3)}^{*}[\mathfrak{p}, v, s, X, L]$. Then, by Definition 8, we see that

$$
\left|\frac{(L-1) \frac{z^{v^{(}\left(\mathfrak{D}_{q}^{(v+s)} f\right)(z)}}{\left(\mathfrak{D}_{q}^{(s)} f\right)(z)}-(X-1)}{(L+1) \frac{z^{v^{(}\left(\mathfrak{D}_{q}^{(\nu+s)} f\right)(z)}}{\left(\mathfrak{D}_{q}^{(s)} f\right)(z)}-(X+1)}-1\right|<1,
$$

so that

$$
\left|\frac{(L-1) \frac{z^{\nu}\left(\mathcal{D}_{q}^{(\nu+s)} f\right)(z)}{\left(\mathfrak{D}_{q}^{(s)} f\right)(z)}-(X-1)}{(L+1) \frac{z^{\nu}\left(\mathfrak{D}_{q}^{(\nu+s)} f\right)(z)}{\left(\mathcal{D}_{q}^{(s)} f\right)(z)}-(X+1)}-1\right|+\frac{q}{1-q}<1+\frac{q}{1-q} .
$$

By applying the triangle inequality in equation (2.1), we get

$$
\left|\frac{(L-1) \frac{z^{\nu}\left(\mathfrak{D}_{q}^{(\nu+s)} f\right)(z)}{\left(\mathfrak{D}_{q}^{(s)} f\right)(z)}-(X-1)}{(L+1) \frac{z^{\nu}\left(\mathfrak{D}_{q}^{(v+s)} f\right)(z)}{\left(\mathfrak{D}_{q}^{(s)} f\right)(z)}-(X+1)}-\frac{1}{1-q}\right|<\frac{1}{1-q} .
$$

The last expression in (2.2) now implies that $f \in \mathcal{S}_{(q, 2)}^{*}[\mathfrak{p}, v, s, X, L]$, that is,

$$
\mathcal{S}_{(q, 3)}^{*}[\mathfrak{p}, v, s, X, L] \subset \mathcal{S}_{(q, 2)}^{*}[\mathfrak{p}, v, s, X, L]
$$

We next suppose $f \in \mathcal{S}_{(q, 2)}^{*}[\mathfrak{p}, v, s, X, L]$, so that

$$
f \in \mathcal{S}_{(q, 2)}^{*}[\mathfrak{p}, v, s, X, L] \Longleftrightarrow\left|\frac{(L-1) \frac{z^{\nu}\left(\mathfrak{D}_{q}^{(v+s)} f\right)(z)}{\left(\mathfrak{D}_{q}^{(s)} f\right)(z)}-(X-1)}{(L+1) \frac{z^{\nu}\left(\mathfrak{D}_{q}^{(q+s)}\right)(z)}{\left(\mathfrak{D}_{q}^{(s)} f\right)(z)}-(X+1)}-\frac{1}{1-q}\right|<\frac{1}{1-q},
$$

by Definition 7 . 
Since

$$
\begin{array}{r}
\frac{1}{1-q}>\left|\frac{(L-1) \frac{z^{\nu}\left(\mathfrak{D}_{q}^{(v+s)} f\right)(z)}{\left(\mathfrak{D}_{q}^{(s)} f\right)(z)}-(X-1)}{(L+1) \frac{z^{v}\left(\mathfrak{D}_{q}^{(v+s)} f\right)(z)}{\left(\mathfrak{D}_{q}^{(s)} f\right)(z)}-(X+1)}-\frac{1}{1-q}\right| \\
=\left|\frac{1}{1-q}-\frac{(L-1) \frac{z^{\nu}\left(\mathfrak{D}_{q}^{(v+s)} f\right)(z)}{\left(\mathfrak{D}_{q}^{(s)} f\right)(z)}-(X-1)}{(L+1) \frac{z^{v}\left(\mathfrak{D}_{q}^{(v+s)} f\right)(z)}{\left(\mathfrak{D}_{q}^{(s)} f\right)(z)}-(X+1)}\right|,
\end{array}
$$

we have

$$
\mathfrak{R}\left(\frac{(L-1) \frac{z^{v}\left(\mathfrak{D}_{q}^{(v+s)} f\right)(z)}{\left(\mathfrak{D}_{q}^{(s)} f\right)(z)}-(X-1)}{(L+1) \frac{z^{v}\left(\mathfrak{D}_{q}^{(v+s)} f\right)(z)}{\left(\mathfrak{D}_{q}^{(s)} f\right)(z)}-(X+1)}\right)>0 \quad(z \in \mathbb{U}) .
$$

This last inequality now shows that $f \in \mathcal{S}_{(q, 1)}^{*}[\mathfrak{p}, v, s, X, L]$, that is,

$$
\mathcal{S}_{(q, 2)}^{*}[\mathfrak{p}, v, s, X, L] \subset \mathcal{S}_{(q, 1)}^{*}[\mathfrak{p}, v, s, X, L]
$$

which completes the proof of Theorem 1.

Remark 2 First of all, if we put $v=s+1=\mathfrak{p}=1$ in Theorem 1, we get the corresponding result due to Srivastava et al. [33]. Secondly, if we put

$$
X=1-2 \alpha \quad(0 \leqq \alpha<1) \quad \text { and } \quad L=-1
$$

Theorem 1 gives the corresponding result that was proved by Khan et al. [8]. Thirdly, if we assign the following values to the parameters in Theorem 1:

$$
X=1-2 \alpha \quad(0 \leqq \alpha<1) \quad \text { and } \quad-L=v=s+1=\mathfrak{p}=1
$$

we get the result which was proved by Wongsaijai and Sukantamala [35].

Corollary 1 (see [35]) For $0 \leqq \alpha<1$,

$$
\mathcal{S}_{q, 3}^{*}(\alpha) \subset \mathcal{S}_{q, 2}^{*}(\alpha) \subset \mathcal{S}_{q, 1}^{*}(\alpha)
$$

Finally, below a sufficient condition for the class $\mathcal{S}_{(q, 3)}^{*}[\mathfrak{p}, v, s, X, L]$ is given, which also includes the corresponding sufficient conditions for the function classes $\mathcal{S}_{(q, 1)}^{*}[\mathfrak{p}, v, s, X, L]$ and $\mathcal{S}_{(q, 2)}^{*}[\mathfrak{p}, v, s, X, L]$.

Theorem 2 A function $f \in \mathcal{A}(\mathfrak{p})$ having form (1.1) is in the class $\mathcal{S}_{(q, 3)}^{*}[\mathfrak{p}, v, s, X, L]$ if it satisfies the following coefficient inequality:

$$
\sum_{n=1}^{\infty}\left(2 \Upsilon_{(2, n)}+\left|\Upsilon_{(3, n)}\right|\right)\left|a_{n+\mathfrak{p}}\right|<\left|\Upsilon_{4}\right|-2 \Upsilon_{1}
$$


where

$$
\begin{aligned}
& \Upsilon_{1}=\left(\frac{[\mathfrak{p}]_{q} !}{[\mathfrak{p}-s-v]_{q} !}-\frac{[\mathfrak{p}]_{q} !}{[\mathfrak{p}-s]_{q} !}\right), \\
& \Upsilon_{(2, n)}=\left(\frac{[n+\mathfrak{p}]_{q} !}{[n+\mathfrak{p}-s-v]_{q} !}-\frac{[n+\mathfrak{p}]_{q} !}{[n+\mathfrak{p}-s]_{q} !}\right), \\
& \Upsilon_{(3, n)}=\left(\frac{(L+1)[n+\mathfrak{p}]_{q} !}{[n+\mathfrak{p}-s-v]_{q} !}-\frac{(X-1)[n+\mathfrak{p}]_{q} !}{[n+\mathfrak{p}-s]_{q} !}\right),
\end{aligned}
$$

and

$$
\Upsilon_{4}=\left(\frac{(L+1)[\mathfrak{p}]_{q} !}{[\mathfrak{p}-s-v]_{q} !}-\frac{(X+1)[\mathfrak{p}]_{q} !}{[\mathfrak{p}-s]_{q} !}\right)
$$

Proof We begin the proof of Theorem 2 by assuming that the condition in (2.3) holds true. Then it is sufficient to show that

$$
\left|\frac{(L-1) \frac{z^{\nu}\left(\mathfrak{D}_{q}^{(\nu+s)} f\right)(z)}{\left(\mathfrak{D}_{q}^{(s)} f\right)(z)}-(X-1)}{(L+1) \frac{z^{\nu}\left(\mathfrak{D}_{q}^{(\nu+s)} f\right)(z)}{\left(\mathfrak{D}_{q}^{(s)} f\right)(z)}-(X+1)}-1\right|<1 .
$$

We have

$$
\begin{aligned}
& \left|\frac{(L-1) \frac{z^{\nu}\left(\mathfrak{D}_{q}^{(v+s)} f\right)(z)}{\left(\mathfrak{D}_{q}^{(s)} f\right)(z)}-(X-1)}{(L+1) \frac{z^{v}\left(\mathfrak{D}_{q}^{(\nu+s)} f\right)(z)}{\left(\mathfrak{D}_{q}^{(s)} f\right)(z)}-(X+1)}-1\right| \\
& \quad=2\left|\frac{z^{v}\left(\mathfrak{D}_{q}^{(v+s)} f\right)(z)-\left(\mathfrak{D}_{q}^{(s)} f\right)(z)}{(L+1)\left(\mathfrak{D}_{q}^{(v+s)} f\right)(z)-(X+1)\left(\mathfrak{D}_{q}^{(s)} f\right)(z)}\right| \\
& \quad=2 \mid \frac{\Upsilon_{1} z^{\mathfrak{p}-s}+\sum_{n=1}^{\infty} \Upsilon_{(2, n)} a_{n+\mathfrak{p}} z^{n+\mathfrak{p}-s}}{\Upsilon_{4} z^{\mathfrak{p}-s}+\sum_{n=1}^{\infty} \Upsilon_{(3, n)} a_{n+\mathfrak{p}} z^{n+\mathfrak{p}-s} \mid} \\
& \quad \leqq \frac{2 \Upsilon_{1}+2 \sum_{n=1}^{\infty} \Upsilon_{(2, n)}\left|a_{n+\mathfrak{p}}\right|}{\left|\Upsilon_{4}\right|-\sum_{n=1}^{\infty}\left|\Upsilon_{(3, n)}\right|\left|a_{n+\mathfrak{p}}\right|} \quad(|z|=1),
\end{aligned}
$$

where $\Upsilon_{1}, \Upsilon_{(2, n)}, \Upsilon_{(3, n)}$, and $\Upsilon_{4}$ are given by (2.4), (2.5), (2.6), and (2.7) respectively. We see that the last expression in (2.8) is bounded above by 1 if

$$
\sum_{n=1}^{\infty}\left(2 \Upsilon_{(2, n)}+\left|\Upsilon_{(3, n)}\right|\right)\left|a_{n+\mathfrak{p}}\right|<\left|\Upsilon_{4}\right|-2 \Upsilon_{1}
$$

which completes the proof of Theorem 2 .

Remark 3 First of all, if we put $v=s+1=\mathfrak{p}=1$ in Theorem 2, we get the corresponding result due to Srivastava et al. [33]. Secondly, if we put

$$
X=1-2 \alpha \quad(0 \leqq \alpha<1) \quad \text { and } \quad L=-1 \text {, }
$$

Theorem 2 is reduced to the known result, which was stated and proved by Khan et al. [8]. 


\section{Analytic functions with negative coefficients}

The main aim of this section is to present some classes of multivalent $q$-starlike functions involving the functions with negative coefficients. A subset of the class $\mathcal{A}(\mathfrak{p})$ which contains all such functions with negative coefficient, that is,

$$
f(z)=z^{\mathfrak{p}}-\sum_{n=1}^{\infty}\left|a_{n+\mathfrak{p}}\right| z^{n+\mathfrak{p}}
$$

will be denoted here by $\mathcal{T}$. We also let

$$
\mathcal{T} \mathcal{S}_{(q, j)}^{*}[\mathfrak{p}, v, s, X, L]:=\mathcal{S}_{(q, j)}^{*}[\mathfrak{p}, v, s, X, L] \cap \mathcal{T} \quad(j=1,2,3)
$$

Theorem 3 If $-1 \leqq L<X \leqq 1$, then

$$
\mathcal{T} \mathcal{S}_{(q, 1)}^{*}[\mathfrak{p}, v, s, X, L] \equiv \mathcal{T} \mathcal{S}_{(q, 2)}^{*}[\mathfrak{p}, v, s, X, L] \equiv \mathcal{T} \mathcal{S}_{(q, 3)}^{*}[\mathfrak{p}, v, s, X, L]
$$

Proof By the virtue of Theorem 1, it suffices to show that

$$
\mathcal{T S}_{(q, 1)}^{*}[\mathfrak{p}, v, s, X, L] \subseteq \mathcal{T} \mathcal{S}_{(q, 3)}^{*}[\mathfrak{p}, v, s, X, L]
$$

Indeed, in the light of Definition 6 for a function $f \in \mathcal{T S}_{(q, 1)}^{*}[\mathfrak{p}, v, s, X, L]$, we have

$$
\Re\left(\frac{(L-1) \frac{z^{\nu}\left(\mathcal{D}_{q}^{(v+s)} f\right)(z)}{\left(\mathfrak{D}_{q}^{(s)} f\right)(z)}-(X-1)}{(L+1) \frac{z^{\nu}\left(\mathfrak{D}_{q}^{(v+s)} f\right)(z)}{\left(\mathfrak{D}_{q}^{(s)} f\right)(z)}-(X+1)}\right) \geqq 0,
$$

so that

$$
\Re\left(\frac{(L-1) \frac{z^{\nu}\left(\mathcal{D}_{q}^{(v+s)} f\right)(z)}{\left(\mathcal{D}_{q}^{(s)} f\right)(z)}-(X-1)}{(L+1) \frac{z^{v}\left(\mathfrak{D}_{q}^{(\nu+s)} f\right)(z)}{\left(\mathcal{D}_{q}^{(s)} f\right)(z)}-(X+1)}-1\right) \geqq-1 .
$$

After some elementary and simple calculations, we deduce that

$$
\Re\left(\frac{2\left[\left(\mathfrak{D}_{q}^{(s)} f\right)(z)-z^{v}\left(\mathfrak{D}_{q}^{(v+s)} f\right)(z)\right]}{(L+1) z^{v}\left(\mathfrak{D}_{q}^{(v+s)} f\right)(z)-(X+1)\left(\mathfrak{D}_{q}^{(s)} f\right)(z)}\right) \geqq-1,
$$

that is,

$$
-2 \Re\left(\frac{\Upsilon_{1} z^{\mathfrak{p}-s}-\sum_{n=1}^{\infty} \Upsilon_{(2, n)} a_{n+\mathfrak{p}} z^{n+\mathfrak{p}-s}}{\Upsilon_{4} z^{\mathfrak{p}-s}-\sum_{n=1}^{\infty} \Upsilon_{(3, n)} a_{n+\mathfrak{p}} z^{n+\mathfrak{p}-s}}\right) \geqq-1 .
$$

If we now choose that $z$ lies on the real axis, then

$$
\frac{z^{v}\left(\mathfrak{D}_{q}^{(v+s)} f\right)(z)}{\left(\mathfrak{D}_{q}^{(s)} f\right)(z)}
$$


assumes real values. In this case, upon letting $z \rightarrow 1$ - along the real line, we get

$$
2 \Upsilon_{1}-\sum_{n=1}^{\infty} 2 \Upsilon_{(2, n)}\left|a_{n+\mathfrak{p}}\right| \geqq-\left|\Upsilon_{4}\right|+\sum_{n=1}^{\infty}\left|\Upsilon_{(3, n)}\right|\left|a_{n+\mathfrak{p}}\right|
$$

where $\Upsilon_{1}, \Upsilon_{(2, n)}, \Upsilon_{(3, n)}$, and $\Upsilon_{4}$ are given by (2.4), (2.5), (2.6), and (2.7) respectively. We see that the last expression in (3.3) satisfies the inequality in (2.3). Hence our proof of Theorem 3 is now completed.

Remark 4 First of all, if we put $v=s+1=\mathfrak{p}=1$ in Theorem 3, we get the corresponding result due to Srivastava et al. [33]. Secondly, if we put

$$
X=1-2 \alpha \quad(0 \leqq \alpha<1) \quad \text { and } \quad L=-1 \text {, }
$$

Theorem 3 gives the corresponding result that was proved by Khan et al. [8]. Thirdly, if we assign the following values to the parameters in Theorem 3:

$$
X=1-2 \alpha \quad(0 \leqq \alpha<1) \quad \text { and } \quad-L=v=s+1=\mathfrak{p}=1,
$$

we have the following known result.

Corollary 2 (see [35, Theorem 8]) If $0 \leqq \alpha<1$, then

$$
\mathcal{T S}_{(q, 1)}^{*}(\alpha) \equiv \mathcal{T} \mathcal{S}_{(q, 2)}^{*}(\alpha) \equiv \mathcal{T} \mathcal{S}_{(q, 3)}^{*}(\alpha)
$$

Corollary 3 Let the function $f$ of the form (3.1) be in the class $\mathcal{T} \mathcal{S}_{q}^{*}[j, \mathfrak{p}, v, s, X, L](j=1,2,3)$.

Then

$$
a_{n+\mathfrak{p}} \leqq \frac{\left|\Upsilon_{4}\right|-2 \Upsilon_{1}}{\left(2 \Upsilon_{(2, n)}+\left|\Upsilon_{(3, n)}\right|\right)}
$$

The following function $f_{t}(z)$

$$
f_{t}(z)=z^{\mathfrak{p}}-\frac{\left|\Upsilon_{4}\right|-2 \Upsilon_{1}}{\left(2 \Upsilon_{(2,1)}+\left|\Upsilon_{(3,1)}\right|\right)} z^{\mathfrak{p}+1}
$$

is best possible, where $\Upsilon_{1}, \Upsilon_{(2, n)}, \Upsilon_{(3, n)}$, and $\Upsilon_{4}$ are given by (2.4), (2.5), (2.6), and (2.7), respectively.

By means of Theorem 3, it should be understood that Type 1, Type 2, and Type 3 of the multivalent $q$-starlike functions which involve the Janowski functions are similar. Therefore, for convenience, we state and prove the following distortion theorem by using the notation $\mathcal{T} \mathcal{S}_{q}^{*}[j, \mathfrak{p}, v, s, X, L]$ in which it is tacitly assumed that $j=1,2,3$.

Theorem 4 If $f \in \mathcal{T S}_{q}^{*}[j, \mathfrak{p}, v, s, X, L](j=1,2,3)$, then

$$
|f(z)| \geqq r^{\mathfrak{p}}-\left(\frac{\left|\Upsilon_{4}\right|-2 \Upsilon_{1}}{\left(2 \Upsilon_{(2,1)}+\left|\Upsilon_{(3,1)}\right|\right)}\right) r^{\mathfrak{p}+1} \quad(n \in \mathbb{N})\left|z^{\mathfrak{p}}\right|=r^{\mathfrak{p}}(0<r<1)
$$


and

$$
|f(z)| \leqq r^{\mathfrak{p}}+\left(\frac{\left|\Upsilon_{4}\right|-2 \Upsilon_{1}}{\left(2 \Upsilon_{(2,1)}+\left|\Upsilon_{(3,1)}\right|\right)}\right) r^{\mathfrak{p}+1} \quad(n \in \mathbb{N})\left|z^{\mathfrak{p}}\right|=r^{\mathfrak{p}}(0<r<1)
$$

The equalities in (3.6) and (3.7) are attained for the function $f(z)$ given by (3.5) and where $\Upsilon_{1}, \Upsilon_{(2, n)}, \Upsilon_{(3, n)}$, and $\Upsilon_{4}$ are given by (2.4), (2.5), (2.6), and (2.7), respectively.

Proof The following inequality can be easily deduced from Theorem 2:

$$
\left(2 \Upsilon_{(2,1)}+\left|\Upsilon_{(3,1)}\right|\right) \sum_{n=1}^{\infty}\left|a_{n+\mathfrak{p}}\right| \leqq \sum_{n=1}^{\infty}\left(2 \Upsilon_{(2, n)}+\left|\Upsilon_{(3, n)}\right|\right)\left|a_{n+\mathfrak{p}}\right|<\left|\Upsilon_{4}\right|-2 \Upsilon_{1}
$$

which yields

$$
|f(z)| \leqq r^{\mathfrak{p}}+\sum_{n=1}^{\infty}\left|a_{n+\mathfrak{p}}\right| r^{n+\mathfrak{p}} \leqq r^{\mathfrak{p}}+r^{\mathfrak{p}+1} \sum_{n=1}^{\infty}\left|a_{n+\mathfrak{p}}\right| \leqq r^{\mathfrak{p}}+\frac{\left|\Upsilon_{4}\right|-2 \Upsilon_{1}}{\left(2 \Upsilon_{(2,1)}+\left|\Upsilon_{(3,1)}\right|\right)} r^{\mathfrak{p}+1}
$$

Similarly, we have

$$
|f(z)| \geq r^{\mathfrak{p}}-\sum_{n=1}^{\infty}\left|a_{n+\mathfrak{p}}\right| r^{n+\mathfrak{p}} \geq r^{\mathfrak{p}}-r^{\mathfrak{p}+1} \sum_{n=1}^{\infty}\left|a_{n+\mathfrak{p}}\right| \geq r^{\mathfrak{p}}-\frac{\left|\Upsilon_{4}\right|-2 \Upsilon_{1}}{\left(2 \Upsilon_{(2,1)}+\left|\Upsilon_{(3,1)}\right|\right)} r^{\mathfrak{p}+1}
$$

where $\Upsilon_{1}, \Upsilon_{(2, n)}, \Upsilon_{(3, n)}$, and $\Upsilon_{4}$ are given by (2.4), (2.5), (2.6), and (2.7), respectively. The proof of Theorem 4 is now completed.

Remark 5 First of all, if we put $v=s+1=\mathfrak{p}=1$ in Theorem 1 , we get the corresponding result due to Srivastava et al. [33]. Secondly, if we put

$$
X=1-2 \alpha \quad(0 \leqq \alpha<1) \quad \text { and } \quad L=-1 \text {, }
$$

Theorem 4 will give the corresponding result that was proved by Khan et al. [8]. Thirdly, if we put

$$
X=1-2 \alpha \quad(0 \leqq \alpha<1) \quad \text { and } \quad-L=v=s+1=\mathfrak{p}=1
$$

in Theorem 4 and let $q \longrightarrow 1-$, we have the following known result.

Corollary 4 (see [23]) If $\in \mathcal{T S}^{*}(\alpha)$, then

$$
r-\left(\frac{1-\alpha}{2-\alpha}\right) r^{2} \leqq|f(z)| \leqq r+\left(\frac{1-\alpha}{2-\alpha}\right) r^{2} \quad(|z|=r(0<r<1))
$$

The next theorem (Theorem 5) can be proven similarly as we proved Theorem 4, so we choose to omit the details involved in our proof of Theorem 5.

Theorem 5 Iff $\in \mathcal{T} \mathcal{S}_{q}^{*}[j, \mathfrak{p}, v, s, X, L](j=1,2,3)$, then

$$
\left|f^{\prime}(z)\right| \geqq \mathfrak{p} r^{\mathfrak{p}-1}-\left(\frac{(\mathfrak{p}+1)\left(\left|\Upsilon_{4}\right|-2 \Upsilon_{1}\right)}{\left(2 \Upsilon_{(2,1)}+\left|\Upsilon_{(3,1)}\right|\right)}\right) r^{\mathfrak{p}} \quad(n \in \mathbb{N})\left|z^{\mathfrak{p}}\right|=r^{\mathfrak{p}}(0<r<1)
$$


and

$$
\left|f^{\prime}(z)\right| \leqq \mathfrak{p} r^{\mathfrak{p}-1}+\left(\frac{(\mathfrak{p}+1)\left(\left|\Upsilon_{4}\right|-2 \Upsilon_{1}\right)}{\left(2 \Upsilon_{(2,1)}+\left|\Upsilon_{(3,1)}\right|\right)}\right) r^{\mathfrak{p}} \quad(n \in \mathbb{N})\left|z^{\mathfrak{p}}\right|=r^{\mathfrak{p}}(0<r<1)
$$

The function $f_{t}(z)$ given by (3.5) is the extremal function.

If in Theorem 5 we take

$$
X=1-2 \alpha \quad(0 \leqq \alpha<1) \quad \text { and } \quad-L=v=s+1=\mathfrak{p}=1
$$

and let $q \longrightarrow 1-$, we get the following known result.

Corollary 5 (see [23]) If $\in \in \mathcal{T S}^{*}(\alpha)$, then

$$
1-\left(\frac{2(1-\alpha)}{2-\alpha}\right) r \leqq\left|f^{\prime}(z)\right| \leqq 1+\left(\frac{2(1-\alpha)}{2-\alpha}\right) r \quad(|z|=r(0<r<1))
$$

Finally, for the class $\mathcal{T} \mathcal{S}_{q}^{*}[j, \mathfrak{p}, v, s, X, L](j=1,2,3)$ of multivalent $q$-starlike functions with negative coefficients, the results related to the radii of close-to-convexity, starlikeness, and convexity are deduced.

Theorem 6 Let $f \in \mathcal{T} \mathcal{S}_{q}^{*}[j, \mathfrak{p}, v, s, X, L](j=1,2,3)$. Then, for $|z| \leqq r_{0}(j, \mathfrak{p}, n, X, L, \chi)$, the function $f$ is $\mathfrak{p}$-valent close-to-convex of order $\chi$ with $(0 \leqq \chi<\mathfrak{p})$, where

$$
r_{0}=\inf _{n \geq 1}\left[\frac{\left(2 \Upsilon_{(2, n)}+\left|\Upsilon_{(3, n)}\right|\right)(\mathfrak{p}-\chi)}{\left(\left|\Upsilon_{4}\right|-2 \Upsilon_{1}\right)(n+\mathfrak{p})}\right]^{\frac{1}{n}}
$$

The function $f_{t}(z)$ given by (3.5) is best possible.

Proof Using Theorem 2 in conjunction with (3.1), for $|z|<r_{0}$, we find that

$$
\left|\frac{f^{\prime}(z)}{z^{\mathfrak{p}-1}}-\mathfrak{p}\right|<\mathfrak{p}-\chi \quad\left(|z| \leqq r_{0}\right)
$$

We have thus completed the proof of Theorem 6.

Remark 6 If we put

$$
X=1-2 \alpha \quad(0 \leqq \alpha<1) \quad \text { and } \quad L=-1
$$

Theorem 6 gives the corresponding result that was proved by Khan et al. [8].

Theorem 7 Let $f \in \mathcal{T} \mathcal{S}_{q}^{*}[j, \mathfrak{p}, v, s, X, L](j=1,2,3)$. Then, for $|z| \leqq r_{1}(j, \mathfrak{p}, n, X, L, \chi)$, the function $f$ is a $\mathfrak{p}$-valent starlike of order $\chi$ with $(0 \leqq \chi<\mathfrak{p})$, where

$$
r_{1}=\inf _{n \geq 1}\left[\frac{\left(2 \Upsilon_{(2, n)}+\left|\Upsilon_{(3, n)}\right|\right)(\mathfrak{p}-\chi)}{\left(\left|\Upsilon_{4}\right|-2 \Upsilon_{1}\right)(n+\mathfrak{p}-\chi)}\right]^{\frac{1}{n}}
$$

The result is sharp for the function $f_{t}(z)$ given by (3.5). 
Proof Using arguments similar to those in the proof of Theorem 6, it can be seen that

$$
\left|\frac{z f^{\prime}(z)}{f(z)}-\mathfrak{p}\right|<\mathfrak{p}-\chi \quad\left(|z| \leqq r_{1}\right)
$$

which evidently proves Theorem 7.

Remark 7 If we put

$$
X=1-2 \alpha \quad(0 \leqq \alpha<1) \quad \text { and } \quad L=-1 \text {, }
$$

Theorem 7 gives the corresponding result that was proved by Khan et al. [8].

Corollary 6 Let $f \in \mathcal{T} \mathcal{S}_{q}^{*}[j, \mathfrak{p}, v, s, X, L](j=1,2,3)$. Then, for $|z| \leqq r_{2}(j, \mathfrak{p}, n, X, L, \chi)$, the function $f$ is a $\mathfrak{p}$-valent convex of order $\chi$ with $(0 \leqq \chi<\mathfrak{p})$ where

$$
r_{2}=\inf _{n \geq 1}\left[\frac{\left(2 \Upsilon_{(2, n)}+\left|\Upsilon_{(3, n)}\right|\right) \mathfrak{p}(\mathfrak{p}-\chi)}{\left(\left|\Upsilon_{4}\right|-2 \Upsilon_{1}\right)(n+\mathfrak{p})(n+\mathfrak{p}-\chi)}\right]^{\frac{1}{n}}
$$

The result is sharp for the function $f_{t}(z)$ given by (3.5).

\section{Concluding remarks and observations}

The works presented in this paper are basically motivated by the well-established usage of the basic (or q-) calculus in the context of geometric function theory. Several subfamilies of multivalently (or, more precisely, $\mathfrak{p}$-valently) $q$-starlike functions in the open unit disk $\mathbb{U}$ have been introduced and investigated here rather systematically. We have also highlighted some known and new facts of our results in form of remarks and corollaries which significantly make the results important.

The usage of basic (or $q^{-}$) series in many diverse areas of mathematics and physics makes it very important. By making use of basic (or $q$-) series some wonderful works have been done (see, for example, [28, pp. 350-351]; see also [9, 10, 15], and [14]). Moreover, as we described in Sect. 1, from Srivastava's observation [25] about the so-called $(p, q)$-calculus, we arrived at the point that indeed the result presented in this paper can be produced for the rather straightforward $(p, q)$-variations.

Acknowledgements

We would like to thank the reviewers for their valuable suggestions and comments.

Funding

None.

Availability of data and materials

Not applicable.

\section{Declarations}

Competing interests

The authors declare that they have no competing interests. 


\section{Author details}

${ }^{1}$ School of Mathematical Sciences and Shanghai Key Laboratory of PMMP, East China Normal University, 500 Dongchuan Road, Shanghai 200241, People's Republic of China. ${ }^{2}$ Department of Mathematics and Statistics, University of Victoria, Victoria, British Columbia V8W 3R4, Canada. ${ }^{3}$ Department of Medical Research, China Medical University Hospital, China Medical University, Taichung 40402, Taiwan, Republic of China. ${ }^{4}$ Department of Mathematics and Informatics, Azerbaijan University, 71 Jeyhun Hajibeyli Street, AZ1007 Baku, Azerbaijan. ${ }^{5}$ Department of Economics, Faculty of Economics Administrative and Social Sciences, Hasan Kalyoncu University, TR-27410 Gaziantep, Turkey. ${ }^{6}$ Department of Mathematics, Abbottabad University of Science and Technology, Abbottabad 22010, Pakistan. ${ }^{7}$ Government Akhtar Nawaz Khan (Shaheed) Degree College KTS, Haripur 22620, Pakistan.

\section{Publisher's Note}

Springer Nature remains neutral with regard to jurisdictional claims in published maps and institutional affiliations.

Received: 22 July 2021 Accepted: 29 September 2021 Published online: 07 October 2021

\section{References}

1. Agarwal, P., Baltaeva, U., Tariboon, J.: Solvability of the boundary-value problem for a third-order linear loaded differential equation with the Caputo fractional derivative. In: Special Functions and Analysis of Differential Equations 2020, pp. 321-334. Chapman and Hall/CRC, Boca Raton (2020)

2. Agarwal, P., Dumitru, B., Quan, C.Y., Shaher, M., Machado, J.A.T.: Fractional calculus ICFDA. In: Conference Proceedings ICFDA, Amman, Jordan, July 16-18, p. 5 (2018)

3. Attiya, A.A., Lashin, A.M., Ali, E., Agarwal, P.: Coefficient bounds for certain classes of analytic functions associated with Faber polynomial. Symmetry 13, Article ID 302 (2021)

4. Ismail, M.E.-H., Merkes, E., Styer, D.: A generalization of starlike functions. Complex Var. Theory Appl. 14, 77-84 (1990)

5. Jackson, F.H.: On q-definite integrals. Q. J. Pure Appl. Math. 41, 193-203 (1910)

6. Jackson, F.H.: q-Difference equations. Am. J. Math. 32, 305-314 (1910)

7. Janowski, W.: Some extremal problems for certain families of analytic functions. Ann. Pol. Math. 28, $297-326$ (1973)

8. Khan, B., Liu, Z.-G., Srivastava, H.M., Khan, N., Darus, M., Tahir, M.: A study of some families of multivalent $q$-starlike functions involving higher-order q-derivatives. Mathematics 8, Article ID 1470 (2020)

9. Khan, B., Srivastava, H.M., Arjika, S., Khan, S., Khan, N., Ahmad, Q.Z.: A certain q-Ruscheweyh type derivative operator and its applications involving multivalent functions. Adv. Differ. Equ. 2021, Article ID 279 (2021)

10. Khan, B., Srivastava, H.M., Khan, N., Darus, M., Ahmad, Q.Z., Tahir, M.: Applications of certain conic domains to a subclass of q-starlike functions associated with the Janowski functions. Symmetry 13, Article ID 574 (2021)

11. Khan, B., Srivastava, H.M., Khan, N., Darus, M., Tahir, M., Ahmad, Q.Z.: Coefficient estimates for a subclass of analytic functions associated with a certain leaf-like domain. Mathematics 8, Article ID 1334 (2020)

12. Khan, B., Srivastava, H.M., Tahir, M., Darus, M., Ahmad, Q.Z., Khan, N.: Applications of a certain integral operator to the subclasses of analytic and bi-univalent functions. AIMS Math. 6, 1024-1039 (2021)

13. Khan, Q., Arif, M., Raza, M., Srivastava, G., Tang, H.: Some applications of a new integral operator in $q$-analog for multivalent functions. Mathematics 7(12), Article ID 1178 (2019)

14. Liu, Z.-G.: Some operator identities and q-series transformation formulas. Discrete Math. 265, 119-139 (2003)

15. Liu, Z.-G.: Two q-difference equations and q-operator identities. J. Differ. Equ. Appl. 16, 1293-1307 (2010)

16. Mahmood, S., Ahmad, Q.Z., Srivastava, H.M., Khan, N., Khan, B., Tahir, M.: A certain subclass of meromorphically q-starlike functions associated with the Janowski functions. J. Inequal. Appl. 2019, Article ID 88 (2019)

17. Mahmood, S., Raza, N., AbuJarad, E.S., Srivastava, G., Srivastava, H.M., Malik, S.N.: Geometric properties of certain classes of analytic functions associated with a q-integral operator. Symmetry 11, Article ID 719 (2019)

18. Mahmood, S., Srivastava, H.M., Khan, N., Ahmad, Q.Z., Khan, B., Ali, l.: Upper bound of the third Hankel determinant for a subclass of q-starlike functions. Symmetry 11, Article ID 347 (2019)

19. Rehman, M.S., Ahmad, Q.Z., Srivastava, H.M., Khan, B., Khan, N.: Partial sums of generalized q-Mittag-Leffler functions. AIMS Math. 5, 408-420 (2019)

20. Rehman, M.S., Ahmad, Q.Z., Srivastava, H.M., Khan, N., Darus, M., Khan, B.: Applications of higher-order q-derivatives to the subclass of $q$-starlike functions associated with the Janowski functions. AlMS Math. 6, 1110-1125 (2021)

21. Ruzhansky, M., Cho, Y.J., Agarwal, P., Area, I. (eds.): Advances in Real and Complex Analysis with Applications Springer, Singapore (2017)

22. Shi, L., Khan, Q., Srivastava, G., Liu, J.-L., Arif, M.: A study of multivalent q-starlike functions connected with circular domain. Mathematics 7, Article ID 670 (2019)

23. Silverman, H.: Univalent functions with negative coefficients. Proc. Am. Math. Soc. 51, 109-116 (1975)

24. Srivastava, H.M.: Univalent functions, fractional calculus, and associated generalized hypergeometric functions. In: Srivastava, H.M., Owa, S. (eds.) Univalent Functions, Fractional Calculus, and Their Applications, pp. 329-354. Halsted, New York (1989)

25. Srivastava, H.M.: Operators of basic (or $q$-) calculus and fractional $q$-calculus and their applications in geometric function theory of complex analysis. Iran. J. Sci. Technol. Trans. A, Sci. 44, 327-344 (2020)

26. Srivastava, H.M., Ahmad, Q.Z., Khan, N., Khan, N., Khan, B.: Hankel and Toeplitz determinants for a subclass of $q$-starlike functions associated with a general conic domain. Mathematics 7, Article ID 181 (2019)

27. Srivastava, H.M., Aouf, M.K., Mostafa, A.O.: Some properties of analytic functions associated with fractional $q$-calculus operators. Miskolc Math. Notes 20, 1245-1260 (2019)

28. Srivastava, H.M., Karlsson, P.W.: Multiple Gaussian Hypergeometric Series. Halsted, New York (1985)

29. Srivastava, H.M., Khan, B., Khan, N., Ahmad, Q.Z.: Coefficient inequalities for q-starlike functions associated with the Janowski functions. Hokkaido Math. J. 48, 407-425 (2019)

30. Srivastava, H.M., Khan, B., Khan, N., Ahmad, Q.Z., Tahir, M.: A generalized conic domain and its applications to certain subclasses of analytic functions. Rocky Mt. J. Math. 49, 2325-2346 (2019) 
31. Srivastava, H.M., Khan, B., Khan, N., Tahir, M., Ahmad, S., Khan, N.: Upper bound of the third Hankel determinant for a subclass of $q$-starlike functions associated with the $q$-exponential function. Bull. Sci. Math. 167, Article ID 102942 (2021)

32. Srivastava, H.M., Raza, N., AbuJarad, E.S.A., Srivastava, G., AbuJarad, M.H.: Fekete-Szegö inequality for classes of $(p, q)$-starlike and $(p, q)$-convex functions. Rev. R. Acad. Cienc. Exactas Fís. Nat., Ser. A Mat. 113, 3563-3584 (2019)

33. Srivastava, H.M., Tahir, M., Khan, B., Ahmad, Q.Z., Khan, N.: Some general classes of $q$-starlike functions associated with the Janowski functions. Symmetry 11, Article ID 292 (2019)

34. Srivastava, H.M., Tahir, M., Khan, B., Ahmad, Q.Z., Khan, N.: Some general families of $q$-starlike functions associated with the Janowski functions. Filomat 33, 2613-2626 (2019)

35. Wongsaijai, L., Sukantamala, N.: Certain properties of some families of generalized starlike functions with respect to q-calculus. Abstr. Appl. Anal. 2016, Article ID 6180140 (2016)

36. Yassen, M.F., Attiya, A.A., Agarwal, P.: Subordination and superordination properties for certain family of analytic functions associated with Mittag-Leffler function. Symmetry 12, Article ID 1724 (2020)

37. Zhou, S.S., Areshi, M., Agarwal, P., Shah, N.A., Chung, J.D., Nonlaopon, K.: Analytical analysis of fractional-order multi-dimensional dispersive partial differential equations. Symmetry 13, Article ID 939 (2021)

\section{Submit your manuscript to a SpringerOpen ${ }^{\circ}$ journal and benefit from:}

- Convenient online submission

- Rigorous peer review

- Open access: articles freely available online

- High visibility within the field

- Retaining the copyright to your article

Submit your next manuscript at $\mathbf{s p r i n g e r o p e n . c o m ~}$ 